\title{
Numerical simulation study on bowtie antenna-based time reversal mirror for super-resolution target detection
}

\author{
Baidenger Agyekum Twumasi ${ }^{* * *}$, Jia-Lin Li ${ }^{*}$
}

\begin{abstract}
Bowtie antenna-based time reversal mirror (TRM), incorporating with randomly distributed and arbitrarily shaped wire metamaterials medium, is proposed to realize super-resolution target detection. The achieved performance for standard and scatterer bowtie antenna TRM is compared and discussed. The dual-band bowtie antennas resonate at $2.45 \mathrm{GHz}$ and $5.2 \mathrm{GHz}$ and a super-resolution of 0.0817 of the free-space wavelength at $2.45 \mathrm{GHz}$ has been achieved. For the first time, studies show that the TRM with microstructure perturbations (namely scatterers) can enhance the resolution in some cases. Proposing a method of super-resolving transmission of electromagnetic waves is very important to realize multi-independent channels in a compact space for the related applications.
\end{abstract}

K e y w ords: electromagnetic time reversal, super-resolution, target detection, time reversal mirror

\section{Introduction}

Time reversal, a common method in wave transmission in inhomogeneous media when a signal is recorded at points of a device known as time reversal mirror (TRM), gets time flipped and retransmitted back into the medium. The resulting field has a focusing property. Basically, time reversal (TR) technique is motion inversion and not time return, which is first in, last out [1].

An innovative technique, which makes use of multipath components in super-resolution focusing is the TR technique, which can improve the detection performance of a radar system in the midst of clutter as well as the signal to noise ratio in a two-way communication system. In a TR process, the medium is illuminated with a probing pulse. The scattered signals are received and recorded by an array of TRM, time reversed and retransmitted into the same medium. As the name suggest, it is like playing a movie backwards. The time-reversed signals propagated backwards through the time autonomous medium will go through the same multiple scattering, reflection and refractions as they went through in the forward transmission, resulting in the focus of energy at the initial source or a scatterer in the medium [2].

The TR technique, introduced in acoustics by M. Fink and others, has opened the door to various applications in electrical engineering and related studies. Typically, multipath is thought to be disadvantageous and undesirable whose effects should be curtailed. That notwithstanding, the TR takes advantage of the multipath effect and manipulates it to constructive use, the more, the better. Electromagnetic TR methods have attracted increasing interest recently with wide applications [3-8] because of focusing waves, both temporally and spatially, through an inhomogeneous medium $[9,10]$. The TR technique was postulated on the reciprocity property of the wave equation and is analogous to phase conjugation in optics. G. Lerosey et al $[11,20]$ proposed an approach of overcoming the diffraction limit with a narrow band TR focusing using some vertical wire array with element spacing of $\lambda / 30$ for far-field time reversal. This has stimulated lots of interest in subwavelength imaging and target localization applications including this contribution [21,22].

To date, most studies about the electromagnetic TR applications are focused on how to excite the fine structure close to the target [22-27] while for the time reversal mirror (TRM) in the far-field, simple or standard probes/sensors are considered [28]. However, our studies show that the TRM is also important to achieve good super-resolution for target detections. In this paper, we primarily discuss the TRM, specifically, bowtie antenna with dual-band operation. Further, micro-structured scatterer bowtie antenna is discussed as the TRM to achieve better resolution. Performance of the standard and scatterer bowtie antennas is comparatively studied. For the first time, we found that the TRM with microstructure perturbations (namely scatterers) can enhance the resolution in some cases.

\section{Design of standard and scatterer based bowtie antennas}

Characterized by light weight, boresight radiation performance and compactness, planar subwavelength dualband bowtie antennas etched periodically with defected

*School of Physics, University of Electronic Science and Technology of China, Chengdu 610054, China **Department of Electrical/Electronic Engineering, Ho Technical University (HTU), Ho, Ghana, btwumasi@htu.edu.gh, jialinli@uestc.edu.cn 


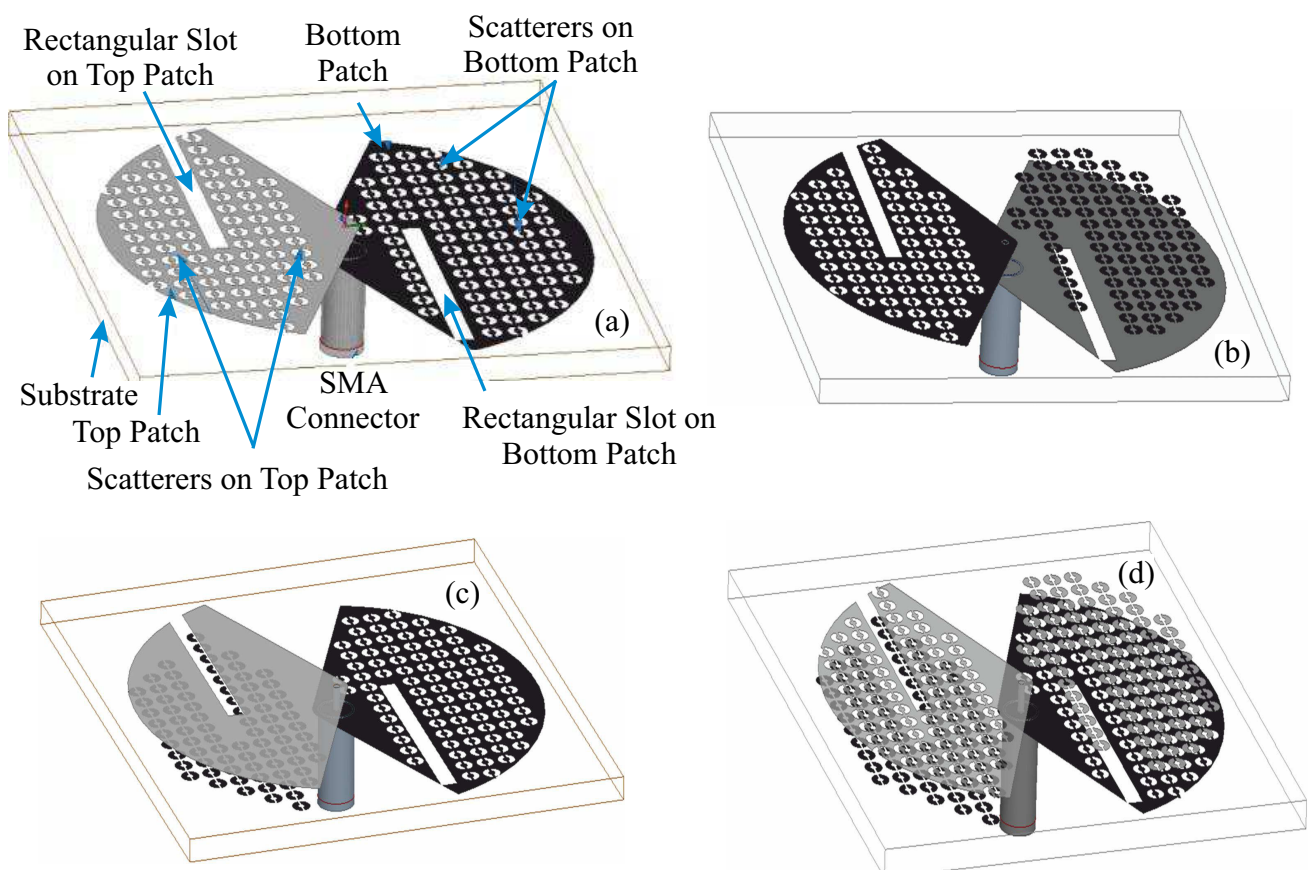

Fig. 1. 3-D view of the dual-band scatterer based bowtie antennas: (a) - scatterers on both patches(type I), (b) - scatterers on top patch and top side of substrate (type II), (c) - scatterers on bottom patch and bottom side of substrate (type III), (d) - scatterers on both patches and both sides of substrate (type IV)
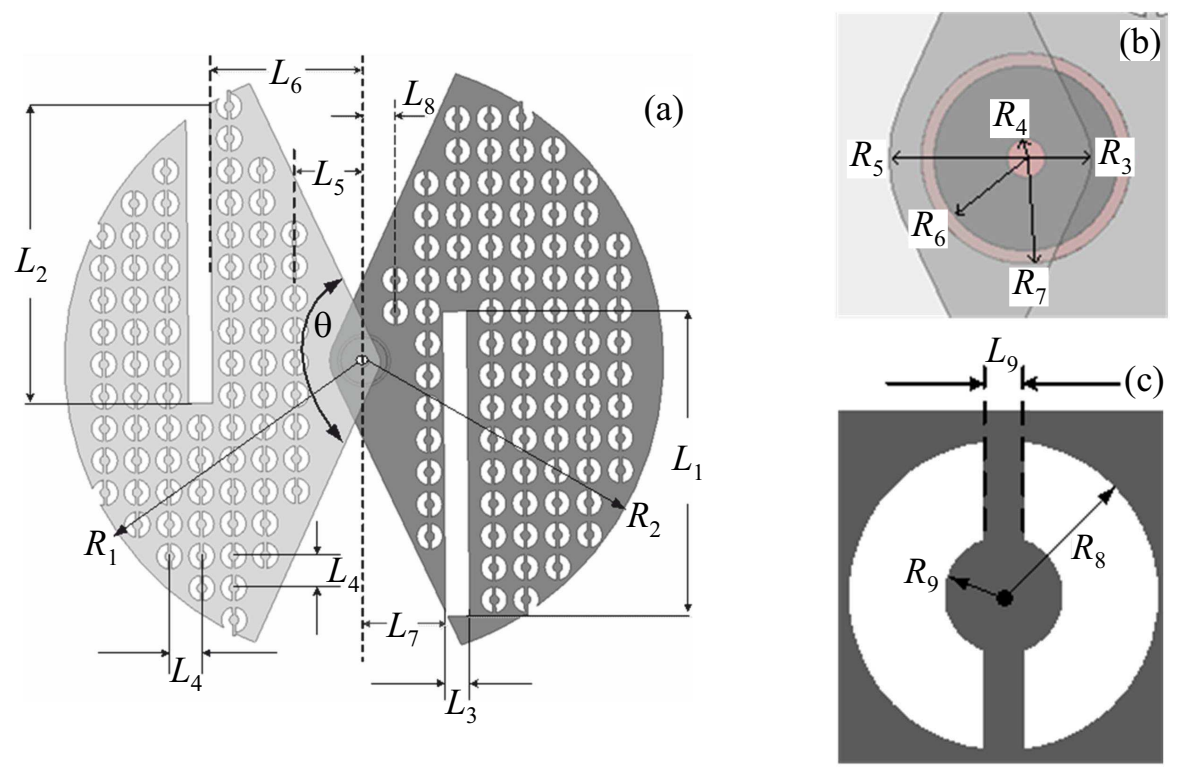

Fig. 2. Dimensions of the proposed scatterer bowtie antenna, where a unit cell of the scatterer is detailed

Table 1. Dimension parameters of dual-band scatterer based Bowtie antenna TRM

\begin{tabular}{lccccc}
\hline Parameter & Unit $(\mathrm{mm})$ & Parameter & Unit $(\mathrm{mm})$ & Parameter & Unit $(\mathrm{mm})$ \\
\hline$L 1$ & 19.0 & $L 7$ & 4.95 & $R 4$ & 0.30 \\
$L 2$ & 18.0 & $L 8$ & 3.69 & $R 5$ & 2.16 \\
$L 3$ & 1.50 & $L 9$ & 0.20 & $R 6$ & 1.45 \\
$L 4$ & 2.0 & $R 1$ & 18.57 & $R 7$ & 1.65 \\
$L 5$ & 4.74 & $R 2$ & 18.70 & $R 8$ & 0.80 \\
$L 6$ & 9.50 & $R 3$ & 1.03 & $R 9$ & 0.30 \\
$\theta$ & $130^{\circ}$ & & & & \\
\hline
\end{tabular}



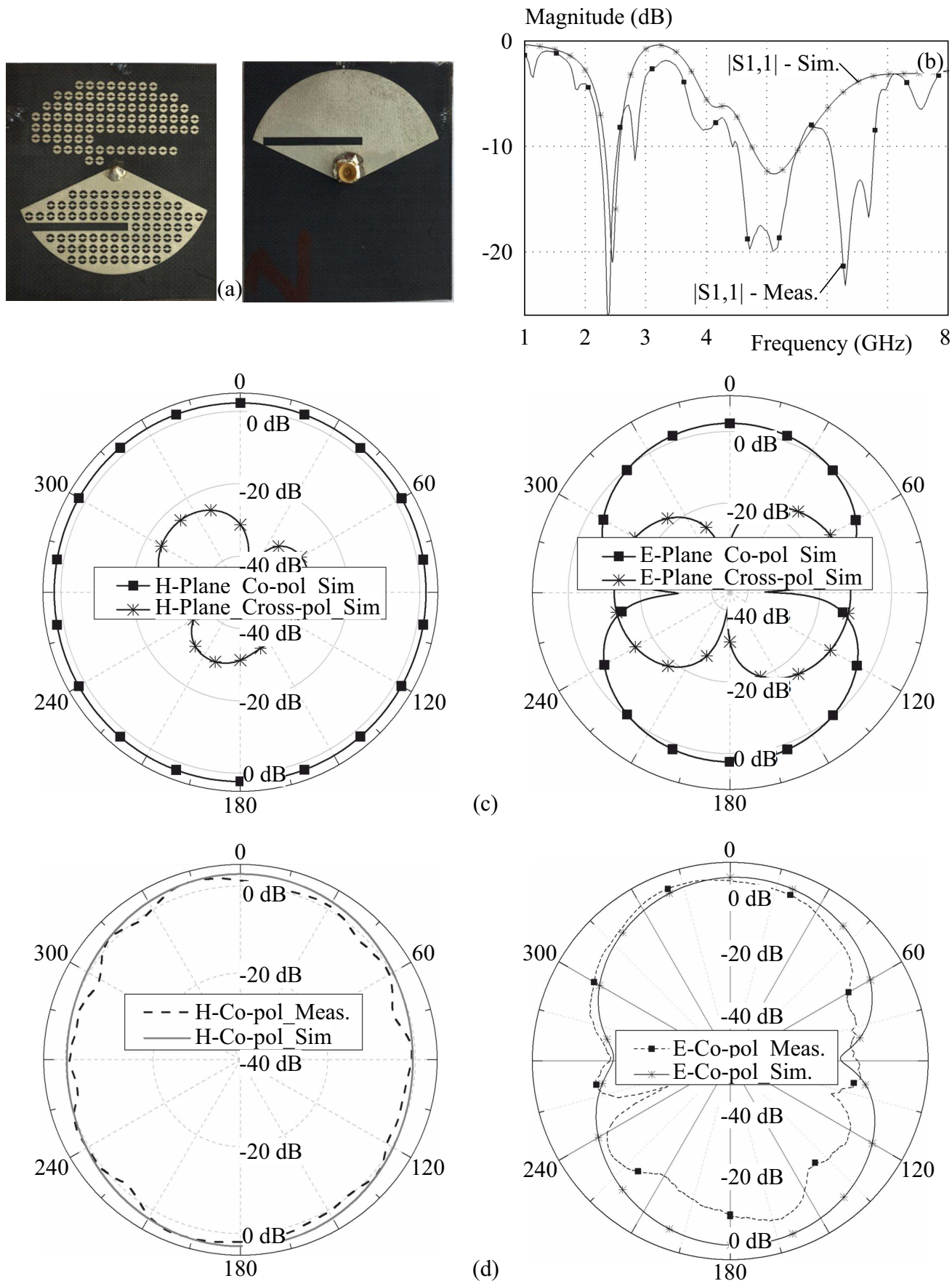

c)

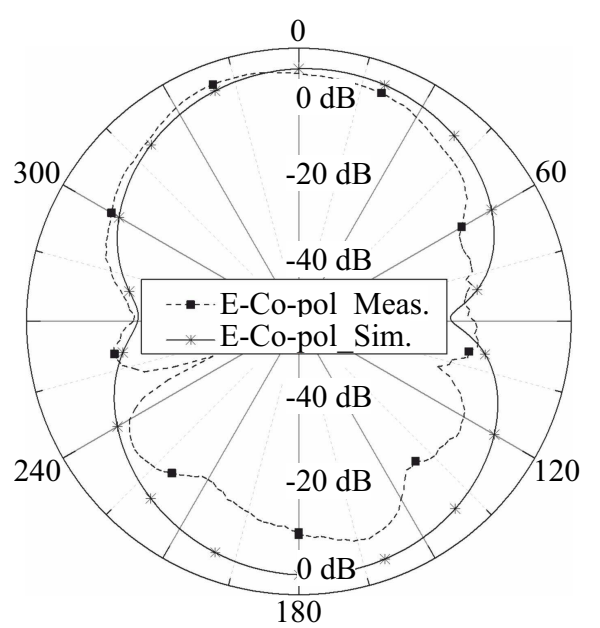

Fig. 3. Performance of the studied dual-band scatterer bowtie antenna: (a) -photographs of the proposed dual-band scatterer based bowtie antenna (type ii), (b) - simulated and measured return losses, (c) - simulated radiation patterns at $2.45 \mathrm{GHz}$, (d) - simulated and measured radiation patterns at $5.2 \mathrm{GHz}$
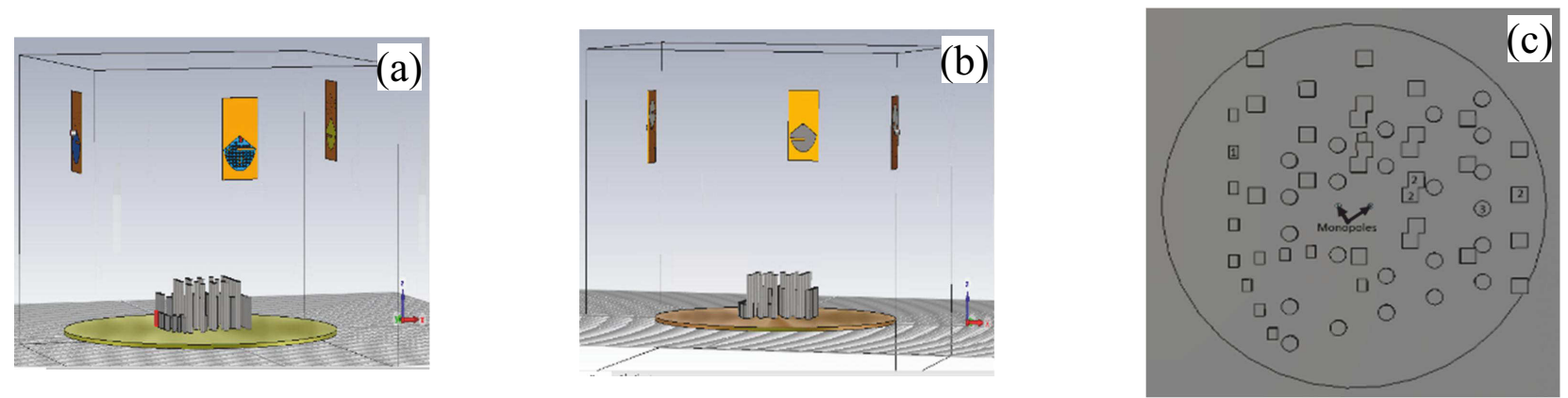

Fig. 4. The numerical simulation setup: (a) - scatterer based bowtie antenna as the TRM, (b) - standard bowtie antenna as the TRM, (c) - the randomly placed wire metamaterial medium 


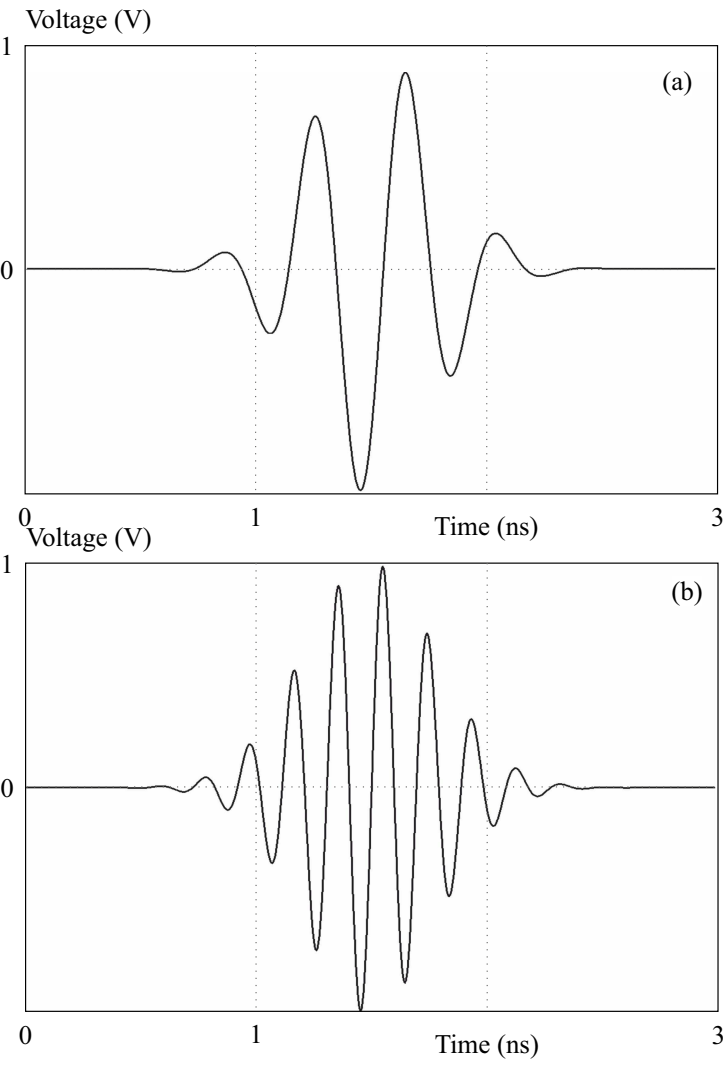

Fig. 5. The ideal Gaussian pulses used in simulations: (a) - at 2.45 $\mathrm{GHz},(\mathrm{b})-$ at $5.2 \mathrm{GHz}$

Table 2. Dimension parameters of vertical scatterers

\begin{tabular}{lccc}
\hline Parameter & Unit $(\mathrm{mm})$ & Parameter & Unit $(\mathrm{mm})$ \\
\hline Cylinder_3 radius & 2.5 & Square_2 $z=h$ & 20 \\
Cylinder_3 height & 30 & Rectangle_1 $x$ & 3 \\
Square_2 $x$ & 5 & Rectangle_1 $y$ & 4 \\
Square_2 $y$ & 5 & Rectangle_3 $z=h$ & 10 \\
\hline
\end{tabular}

scatterers on the radiators and substrate for superresolution focusing with far-field time reversal incorporating with wire metamaterials medium is proposed here as TRM. It is found that majority of the reported subwavelength arrays such as [16-22,27] were all wide-band to ultra-wide-band antenna designs to the best of our knowledge. In this work, a dual-band bowtie antenna [26] referred as the basic structure is first considered. The dual-band operation is specified as $2.45 \mathrm{GHz}$ and $5.2 \mathrm{GHz}$ for potential industrial-scientific-medical (ISM) band applications. Details of the standard dual-band bowtie antenna can be found in [26].

The scatterer bowtie antennas, based on the basic structure for dual-band operations, have been designed, fabricated and characterized, as shown in Fig. 1(a)-(d) with details shown in Fig. 2(a)-(c). As can be seen from Fig. 1, the split-ring microstructures were etched on various sides of the top and bottom patches and the substrate, giving us four different variations of the dual band antennas designed with very similar characteristics. All the four scatterer based dual-band bowtie antennas were fabricated on a substrate with a thickness of $0.8 \mathrm{~mm}$, a relative permittivity of 2.65 and a loss tangent of 0.003 . The substrate dimensions are $48.9 \mathrm{~mm}$ by $38.8 \mathrm{~mm}$. Details of the dimension parameters are listed in Tab. 1.

Photographs of one of the fabricated proposed dualband bowtie antenna (type II) are shown in Fig. 3(a). The simulated and measured return losses are shown in Fig. 3(b). From Fig. 3(b), the impedance bandwidth of the dual band antenna referred to $\left|S_{1,1}\right| \leq-10 \mathrm{~dB}$ are: $2.26 \mathrm{GHz}$ to $2.51 \mathrm{GHz}$ for the lower band and $4.54 \mathrm{GHz}$ to $5.52 \mathrm{GHz}$ for the upper band from measurements, while $2.32 \mathrm{GHz}$ to $2.56 \mathrm{GHz}$ for resonance at $2.45 \mathrm{GHz}$ and $4.75 \mathrm{GHz}$ to $5.51 \mathrm{GHz}$ for resonance at $5.2 \mathrm{GHz}$ from simulations. Figures. 3(c) and 3(d) present the radiation patterns at the two resonances, where Fig. 3(c) shows only the simulated radiation patterns at $2.45 \mathrm{GHz}$ due to the lack of measuring horns currently. Figure $3(\mathrm{~d})$ shows the measured and simulated radiation patterns at $5.2 \mathrm{GHz}$. The simulated and measured radiation patterns are in reasonable agreement with a slight discrepancy, where the slight difference in the backside radiation pattern for the E-plane may be due to the reflections from the coaxial cable and mounting fixtures used in the measurements.

\section{Super-resolution characterization of the dual-band standard and scatterer bowtie antennas}

Achieving any resolution better than the Rayleigh resolution or imaging beyond the diffraction limit is referred to as super resolution. Now, the above developed dualband bowtie antennas are further served as the TRM for super resolution applications. For performance comparisons, here both standard and scatterer based bowtie antennas are simulated and numerically verified using CST simulation software. The simulation scenarios are shown in Fig. 4(a)-(c) with the wire metamaterial medium.

Figures 4(a) and (b) show the setup of the experiment. Fig. 4(c) shows the close-up view of the layout of the monopoles and the distribution of the near-field arbitrary scatterers. The TRM bowtie were placed $150 \mathrm{~mm}$ from the monopoles and at a height of $80 \mathrm{~mm}$ from the ground plane as shown in Fig. 4(a) and (b). The distance of $150 \mathrm{~mm}$ was the experimentally determined far-field distance of this scatterer-based dual-band bowtie antennas and the tightly coupled monopoles in the setup shown in Fig. 4(a). This distance can also be calculated numerically as $|\gamma| D^{2} / \pi$, where $\gamma$ is the propagation constant of the medium, or at a distance greater than $2 D^{2} / \lambda$, where $D$ is the largest dimension of the antenna for far-field observation and $\lambda$ is the operation wavelength. A total of 57 arbitrary shaped wire metamaterials were used, numbered as shown in Fig. 4c and its detailed dimension parameters are listed in Tab. 2. An initial probing pulse from one of 

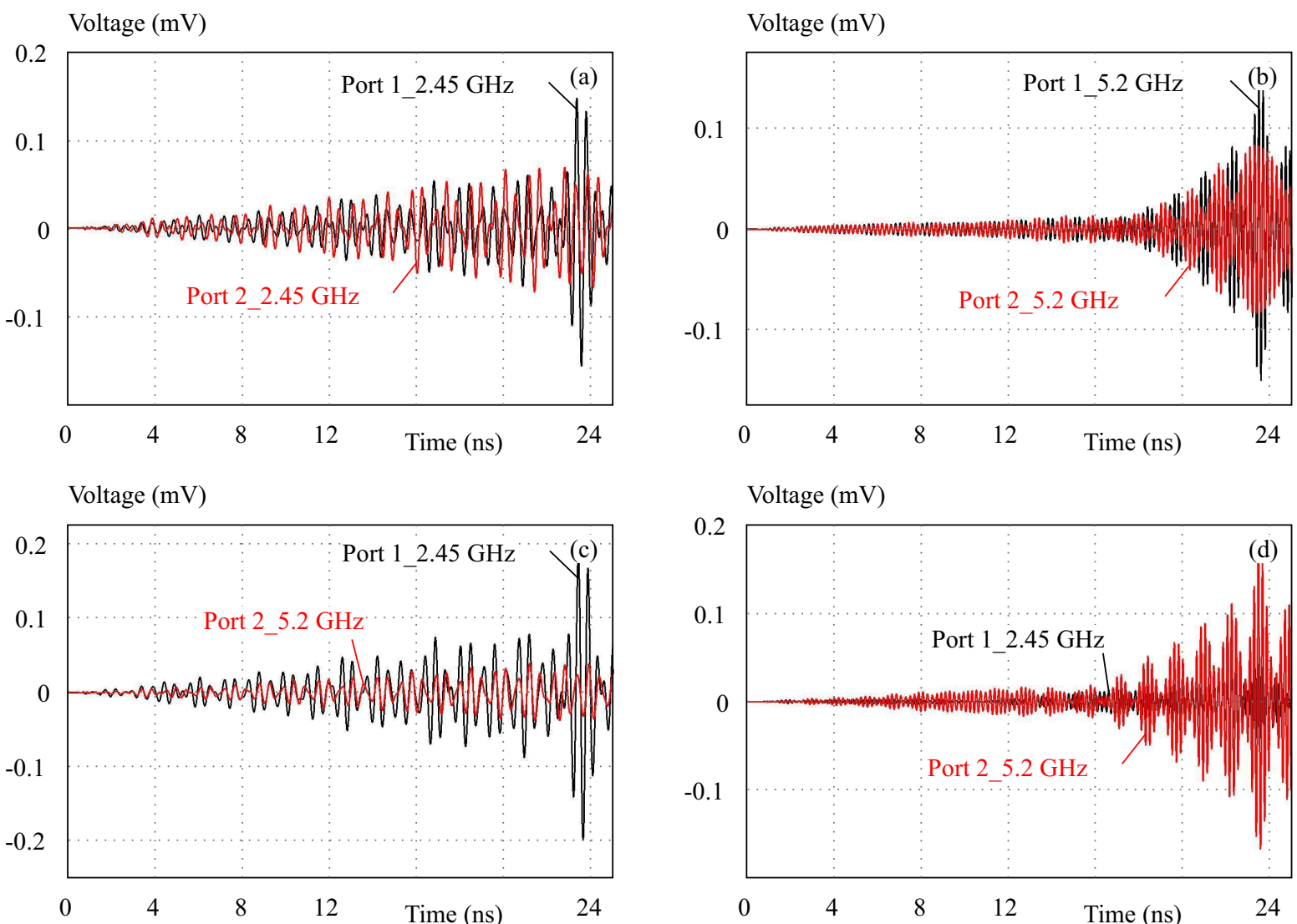

Fig. 6. Super-resolution performance of the SCATTERER based bowtie antenna TRM incorporating with wire metamaterials: (a) port 1 excitation, where two monopoles are of the same height and resonating at $2.45 \mathrm{GHz}$, (b) - port 1 excitation, where two monopoles are of the same height and resonating at $5.2 \mathrm{GHz}$, (c) - port 1 excitation corresponding to the monopole resonating at $2.45 \mathrm{GHz}$, but another monopole has a resonance of $5.2 \mathrm{GHz},(\mathrm{d})$ - port 2 excitation corresponding to the monopole resonating at $5.2 \mathrm{GHz}$, while the other monopole has a resonance of $2.45 \mathrm{GHz}$
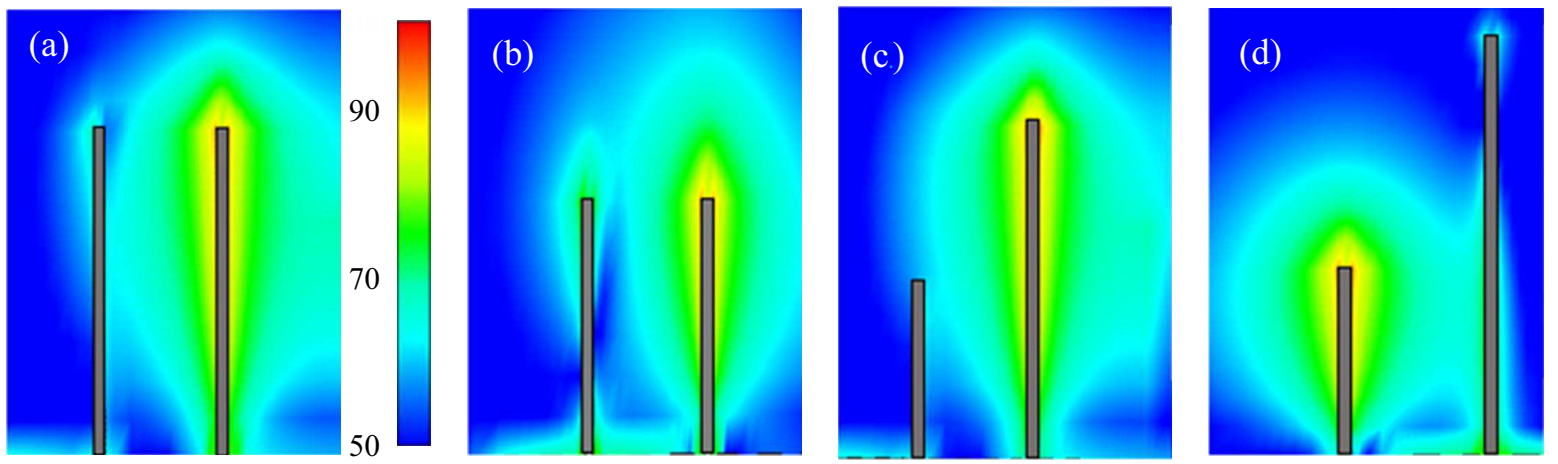

Fig. 7. Typical electric fields of the scatterer based bowtie antenna TRM incorporating with wire metamaterials corresponding to Fig. 6(a)-(d)

the two monopoles (with a separation of $10 \mathrm{~mm}$ ) is transmitted into the detection domain, and signals received by the TRM were recorded and time reversed by a computer with an in-house MATLAB code and re-transmitted back into the detection domain for each test frequencies. The same procedure was repeated with the scatterer bowtie TRM for performance comparisons. The excitation pulse was an amplitude modulated Gaussian pulse with a duration of 3 ns centered at $2.45 \mathrm{GHz}$ and $5.2 \mathrm{GHz}$, as shown in Fig. 5.
The final detection signal received by the respective monopoles focused on the $j^{\text {th }}$ element, denoting a time reversed transmitted signal. The signal received at the $j^{\text {th }}$ monopole and electric field distributions were observed for super resolution characterizations. In our numerical experiments, we outlined two super resolution conditions to be met simultaneously as follows from Tu et al $[23,24]$ :

- After the time reversed transmission, the peak signal amplitude of the channel response from the antenna 

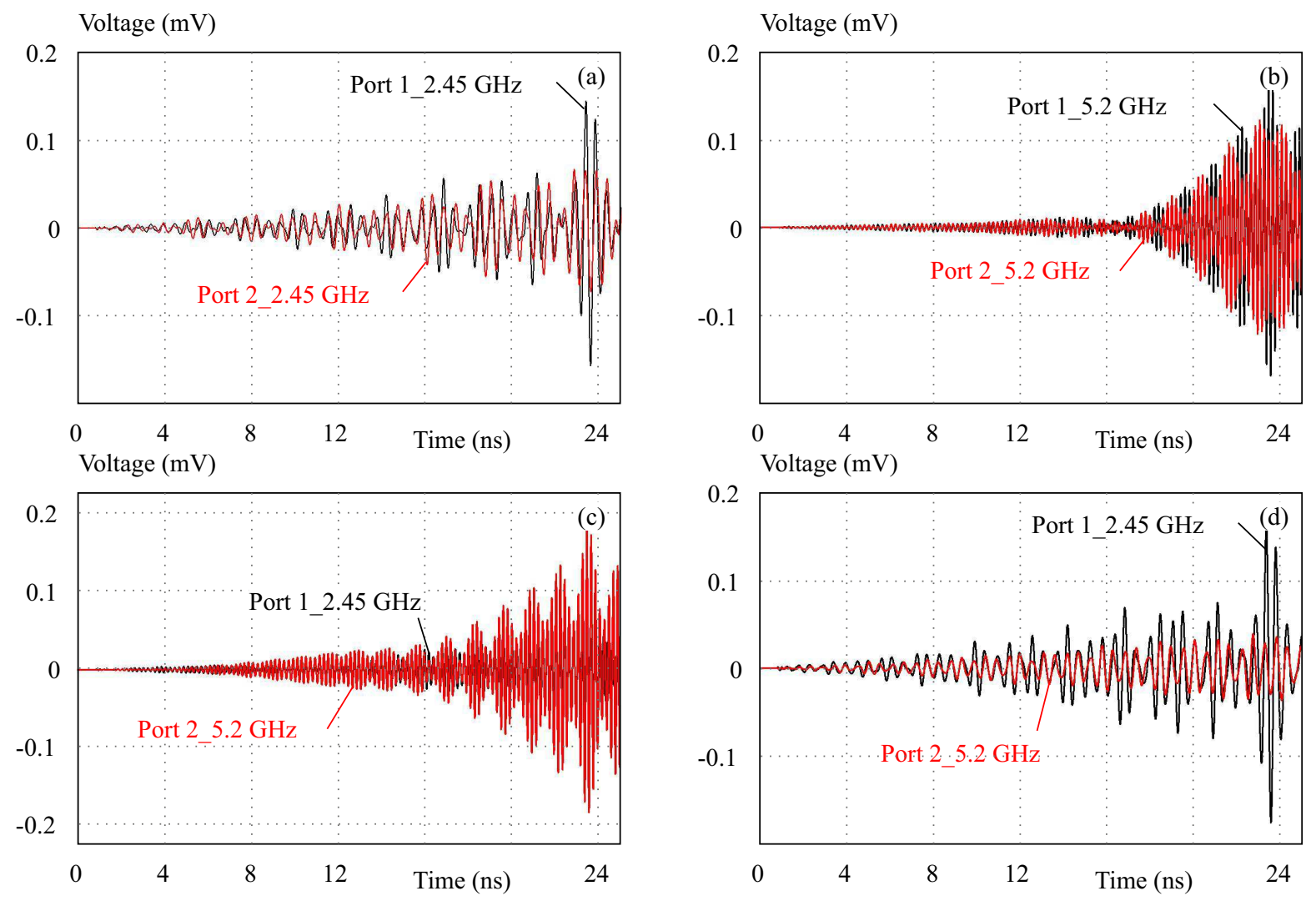

Fig. 8. Super-resolution performance of the STANDARD bowtie antenna TRM incorporating with wire metamaterials: (a) - port 1 excitation, where two monopoles are of the same height and resonating at $2.45 \mathrm{GHz}$; (b) - port 1 excitation, where two monopoles are of the same height and resonating at $5.2 \mathrm{GHz},(\mathrm{c})$ - port 1 excitation corresponding to the monopole resonating at $2.45 \mathrm{GHz}$, but another monopole has a resonance of $5.2 \mathrm{GHz},(\mathrm{d})$ - port 2 excitation corresponding to the monopole resonating at $5.2 \mathrm{GHz}$, while the other monopole has a resonance of $2.45 \mathrm{GHz}$

that transmitted the initial probing signal before the TR phase should be greater by at least 50 percent of the amplitude of the other non-transmitting antennas and should be clearly distinguishable.

- The magnitude of the field distribution of the source transmitting antenna before the TR phase should show a strong electric field distribution and should be clearly distinguishable than the others after TR phase. This implies the energy of the signal could also be considered as a criterion for the observation of superresolution and for our simulation studies, we observed these two criteria for super-resolution.

Figures 6(a)-(d) show the time signal plots of the time reversed transmissions received from the two tightly coupled monopoles labelled as port 1 and 2 after the TR phase under the four frequency scenarios investigated, where ports 1 and 2 refers to the monopole 1 and 2 respectively in all cases.

From Fig. 6, here again, monopoles 1 and 2 with their corresponding frequencies of operation indicated on the graphs. We can observe from Fig. 6(a), monopoles 1 and 2 are operating at the same frequencies but monopole 1 transmitted the initial probing signal before TR and after TR, it is observed that the signal amplitudes from the two tightly coupled monopoles compared indicates that monopole 1 has a peak signal amplitude 50 percent far more than monopole 2 [22]. Similar trend can be observed from Fig. 6(b)-(d) with their corresponding electric field plots in Fig. 7(a)-(d) clearly indicating the realization of super-resolution of the scatterer bowtie antenna at the respective frequencies. Fig. $6(\mathrm{a})-(\mathrm{d})$ also show that TR focusing offers a gain in amplitude of the time signals proportional to the reverberation time of the clutter medium. Further, Fig. 8(a)-(d) show the super-resolution performance of the standard dual-band bowtie antenna TRM [26] without scatterers inclusion where it failed to achieve super resolution at $5.2 \mathrm{GHz}$ as shown in Fig. 8(b). The random distribution of arbitrary scatterers influences the transmitting characteristics and performance of the selected frequencies of transmission rather than the frequency band with super-resolution performance.

From Fig. 6(a)-(d), 7(a)-(d) and 8(a)-(d), we can observe that the scatterer dual-band bowtie TRM incorporating with the wire metamaterial medium has achieved supper-resolution in all the four frequency scenarios compared while the standard bowtie failed at $5.2 \mathrm{GHz}$. With a separation of $10 \mathrm{~mm}$ between the two monopoles, it is found that a super-resolution of $0.0817 \times$ the freespace wavelength at $2.45 \mathrm{GHz}$ is observed. The observed super-resolution phenomenon is due to the resonance phenomenon between the tightly coupled monopoles and the arbitrary shaped metamaterials in the near-field of the 
target which was further enhanced by the scatterers inclusion on the TRM as demonstrated by the simulation results. Moreover, it is believed that the introduced scatterers of the TRM could enhance the near field to be converted to the far field and therefore, this increases the radiation efficiency of the TRM, and finally, enhancing the strength of the received signal for the focusing monopole thus further improving the resolution.

\section{Conclusions}

A scatterer based dual-band bowtie antenna TRM incorporating with arbitrary shaped wire metamaterials medium have been proposed and numerically experimented and compared with a standard bowtie TRM in a time reversal target detection scheme. The dual-band bowtie scatterer based antenna TRM with scatterers inclusion at resonance frequencies of $2.45 \mathrm{GHz}$ and $5.2 \mathrm{GHz}$ has out-performed the standard dual-band bowtie antenna TRM at $5.2 \mathrm{GHz}$ when both monopoles resonate at $5.2 \mathrm{GHz}$ as compared. It is, for the first time, studied the TRM with microstructure perturbations (namely scatterers) and found the resolution can be further enhanced in some cases. Based on the studies, we have demonstrated the realization of super-resolution focusing of $0.0817 \times$ the free-space wavelength at $2.45 \mathrm{GHz}$ on a particular target at dual-band frequencies in the ISM band in this contribution.

\section{REFERENCES}

[1] J. L. Li, B. Z. Wang, D. S. Zhao, and S. Ding, "Recent Researches on Electromagnetic Time Reversal in UESTC", IEEE iWEM - IEEE International Workshop on Electromagnetics: Applications Student Innovation Competition, pp. 16-19, 2013.

[2] M. Fink, "Time-Reversed Acoustics", Scientific American, pp. 91-97, Nov 1999

[3] B. Z. Wang, R. Wang, Z. S. Gong, Q. Gao, and X. H. Wang, "Researches on Far-Field Super-Resolution Imaging Based on Time-Reversed Electromagnetics at UESTC", in IEEE International Conference on Computational Electromagnetics (2016 ICCEM), pp. 319-321, 2016.

[4] E. A. Marengo and J. Tu, "Breast Cancer Detection Based on Time Reversal the Optical Theorem", in Medical Imaging: Ultrasonic Imaging Tomography, p. 941915, March 2015.

[5] Y. Chen, E. Gunawan, K. S. Low, S. W. S. Wang, Y. K. Y. Kim, and C. B. S. Soh, "Pulse Design for Time Reversal Method as Applied to Ultra-Wideband Microwave Breast Cancer Detection: A Two-Dimensional Analysis", IEEE Transactions on Antennas Propagation vol. 55, no, 1, pp. 194-204, 2007.

[6] A. Dezfooliyan and A. M. Weiner, "Experimental Investigation of UWB Impulse Response Time Reversal Technique up to 12 GHz: Omnidirectional Directional Antennas", IEEE Transactions on Antennas Propagation vol. 60, no, 7, pp. 3407-3415, 2012.

[7] P. Kosmas, E. Zastrow, S. C. Hagness, and B. Van Veen, "A Computational Study of Time Reversal Techniques for Ultra-Wideband Microwave Hyperthemia Treatment of Breast Cancer", in IEEE/SP 14th Workshop on Statistical Signal Processing pp. 312-316, Aug 2007.
[8] S. Mukherjee, Y. Deng, L. Udpa, S. Udpa, P. Chahal, and E. Rothwell, "Microwave Time Reversal Mirror for Breast Tumor Detection", in IEEE International Symposium on Antennas Propagation Society (AP-S), pp. 791-792, 2017.

[9] W. M. Dyab, T. K. Sarkar, and M. Salazar-palma, "Antenna Reciprocity the Theory of Electromagnetic Time Reversal", in IEEE International Symposium on Antennas Propagation Society (AP-S), pp. 7-8, 2012.

10] W. G. Dyab, T. K. Sarkar, and M. Salazar-palma, "What is Time Reversal what it Cannot Do?", in IEEE Conference on Antenna Measurements Applications, pp. 1-3, 2014.

[11] J. De Rosny, G. Lerosey, and M. Fink, "Theory of Electromagnetic Time-Reversal Mirrors", IEEE Transactions on Antennas Propagations vol. 58, no, 10, pp. 3139-3149, 2010.

[12] R. J. Potton, "Reciprocity in Optics", Reports on Progress in Physics vol. 67, no, 5, pp. 717-754, 2004.

[13] G. D. Ge, D. Wang, and B. Z. Wang, "Subwavelength Array of Planar Triangle Monopoles with Cross Slots Based on Far-Field Time Reversal", Progress in Electromagnetics Research vol. 114, pp. 429-441, 2011.

14] S. Ding, B. Z. Wang, G. Ge, and D. Zhao, "Sub-Wavelength Array with Embedded Chirped Delay Lines Bsed on Time Reversal Technique", IEEE Transactions on Antennas Propagations vol. 61 , no, 5 , pp. 2868-2873, 2013.

[15] C. A. Valagiannopoulos, "Electromagnetic Scattering of the Field of a Metamaterial Slab Antenna by an Arbitrarily Positioned Cluster of Metallic Cylinders", Progress in Electromagnetics Research vol. 114, pp. 51-66, July 2011.

16] Q. Gao, X. H. Wang, and B. Z. Wang, "Far-Field Sub-Wavelength Imaging of Two-Dimensional Extended Target Aided by Compact Planar Resonant Structures", IEEE Photonics Journal vol. 10, no. 1, Feb 2018.

17] Z. M. Zhang, B. Z. Wang, G. D. Ge, M. S. Liang, and S. Ding, "Sub-Wavelength Spatial Focusing Property of Time Reversal Electromagnetic Wave in Periodic Metal Wire Array", in International Conference on Microwave Millimeter Wave Technology (ICMMT) vol. 4, no, 2, pp. 1330-1332, 2012.

[18] C. Yang, M. Zhu, C. Zhou, and D. Zhao, "Sub-Wavelength UWB Antenna Array with Fractal Slots Split Ring Resonators for Time Reversal Super-Resolution Focusing", in IEEE International Conference on Communication Problem-Solving, pp. 173-175, 2014.

19] R. Wang, B. Z. Wang, Z. S. Gong, and X. Ding, "Far-Field Subwavelength Imaging with Near-Field Resonant Metalens Scanning at Microwave Frequencies", Scientific Report vol. 5, pp. 1035-1036, 2015.

20] A. Wahab, A. Rasheed, T. Hayat, and R. Nawaz, "Electromagnetic Time Reversal Algorithms Source Localization in Lossy Dielectric Media", Communications in Theoretical Physics vol. 62, no, 6, pp. 779-789, 2014.

[21] W. J. R. Hoefer, "Super Resolution Imaging by Computational Time Reversal in Scattering Media", in IEEE International Symposium on Antennas Propagation Society (AP-S), pp. 1302-1303, 2015

22] R. Zhang, W. Shao, and X. Wei, "Sub-Wavelength UWB Antenna Array Design for Super-Resolution", in Progress in Electromagnetic Research Symposium, pp. 4688, 2016.

[23] H. Tu, S. X. Xiao, D. Lesselier, and M. Serhir, "Super-Resolution Characteristics Based on Time-Reversed Single-Frequency Electromagnetic Wave", Journal of Electromagnetic Waves Applications vol. 30, no, 13, pp, 1670-1680, Sep 2016.

24] H. Tu, S. Xiao, J. Xiong, and B. Wang, "Super-Resolution Frequency Spectrum Characteristics of Micro-Structured Array Based on Time Reversal Electromagnetic Wave", in Proceedings of the International Symposium on Antennas \& Propagation (ISAP) vol. 2, pp. 775-778, Oct 2013.

[25] F. Lemoult, G. Lerosey, J. De Rosny, and M. Fink, "Resonant Metalenses for Breaking the Diffraction Barrier", Physical Review Letters vol. 203901, pp. 1-4, May 2010. 
[26] B. A. Twumasi and L. Jia-lin, "Bowtie Antenna TRM Design for Biomedical Imaging Using Electromagnetic Time Reversal Technique", in International Conference on Microwave Millimeter Wave Technology vol. 1, no, 1, pp, 60-62, May 2018.

[27] W. B.-Z. and Z. R. Huang Hai-Yan, Ding Shuai, "Split-RingBased Metamaterial for Far-Field Subwavelength Focusing Based on Time Reversal", Chinese Physics B vol. 23, no. 6, 2014.

[28] S. Mukherjee, Y. Deng, L. Udpa, S. Udpa, P. Chahal, and E. Rothwell, "Microwave Time Reversal Mirror for Breast Tumor Detection", in IEEE International Symposium on Antennas Propagation Society International Symposium (AP-S), pp. 791-792, Oct 2017.

Received 28 March 2019

Baidenger Agyekum Twumasi was born in September, 1981 at Nsawam in the Eastern Region of Ghana. He obtained the HND in Elect/Electronic Engineering from Ho Polytechnic in 2004, the Master of Telecommunication Management from HAN University of Applied Sciences in Netherlands in 2011. He is currently a $\mathrm{PhD}$ candidate in the University of Electronic Science and Technology of China, majoring in Electronic Science and Technology in the School of Physics. He is a lecturer in Elect/Electronic Engineering at Ho Technical University in Ghana. He is also a graduate student member of IEEE and a member of APS and MTTS societies. His research interest includes MIMO Antenna Designs, Planar electronic circuits for Telecommunication Applications, Study of Electromagnetic Time Reversal (EMTR) and its Applications, Wireless Power Transfer and its related circuits, Microwave and Millimeter-wave electronic circuits and systems design as well as Telecommunications Management.

Jia-Lin Li was born in October 1972 at Sichuan, China. He received the MS degree from the University of Electronic Science and Technology of China (UESTC), Chengdu, China, in 2004, and the PhD degree from the City University of Hong Kong, Hong Kong, in 2009, both in electronic engineering. From September 2005 to August 2006, he was a Research Associate with the Wireless Communication Research Center, City University of Hong Kong, Hong Kong. Since September 2009, he has been with the School of Physical Electronics, UESTC, where he is currently a Professor. His research interests include microwave/ millimeter-wave antenna and arrays, circuits and systems, interactions between microwave and complex medium, and so on. 